\title{
Power Quality Real-time Monitoring System Based on WSNs
}

\author{
Yongjun $\mathrm{Yu}^{1,}$, Naifeng Liang ${ }^{2}$, Yingjie Zhang ${ }^{3}$, Zhongyou $\mathrm{Luo}^{4}, \mathrm{Ke} \mathrm{Du}^{5, \mathrm{~b}}$ \\ ${ }^{1,4}$ State Grid Xinjiang Electric Power Research Institute, Urumqi, 830011, China \\ ${ }^{2,3}$ State Grid Xinjiang Electric Power Company, Urumqi, 830000, China \\ ${ }^{5}$ PONOVO Power CO., LTD, Beijing, 100098, China \\ aemail:yujyuj@vip.sina.com;13999347486@163.com;hj9929@sina.com;luozhongyou@126.com, b \\ email:duk@ponovo.cn
}

Keywords: power quality, wireless sensor networks, real-time monitoring system, Zigbee.

\begin{abstract}
With the development of industrial and information technology, the power users increasingly demand better power quality. Therefore, the power department needs to monitor power quality in real time, and timely take appropriate measures to adjust the power quality. To overcome the shortcomings of wired monitoring networks, based on wireless sensor networks technology, this paper presents a real-time monitoring system scheme. Experimental results show that the system is capable to remotely real-time monitor the grid power quality and conveniently provide reliable basis for optimizing grid operation.
\end{abstract}

\section{Introduction}

In the information society, with the extensive application of digital technology, the increasing number of high-precision power electronic equipment is becoming more sensitive to power quality [1]. These fluctuations of voltage and frequency will generate information systems serious consequences, even fatal effects on quality of industrial products. Moreover, with the power electronics technology continues to mature, some high-power, non-linear devices increasingly put into use, making the power quality is deteriorating. Therefore, the design, establishment and optimization of power quality monitoring system have become an important way to ensure the rational and healthy development of the power grid [2-4].

Currently, most of the power quality monitoring systems use wired communication mode for data transmission between data acquisition unit and monitoring center. Taking into account the impact of the geographical environments, electric network composition and construction capabilities, it is not easy to carry out on-line power quality monitoring. The field of wireless sensor networks (WSNs) is receiving much attention in the networking research community and as an interdisciplinary field of interest. It is playing an important role in the next-generation broadband wireless mobile communication networks [5-6]. Consequently, the emergence of WSNs can effectively solve those above-mentioned problems. This paper presents a real-time power quality monitoring system based on WSNs, in which wireless sensor nodes are elaborately placed in the appropriate power monitoring locations. The monitoring center can remotely monitor the power quality indices of each node real time and accurately. The system is characterized by convenient networking, dynamic topology, low cost, high capacity and high reliability.

\section{Power Quality Indices}

The power quality determines the fitness of electric power to all kinds of consumer devices. The synchronization of voltage frequency and phase allows electrical systems to function in their intended manner without significant loss of performance or life [7]. Without the proper power, an electrical device (or load) may malfunction, fail prematurely or not operate at all. In terms of grid operation modes, the current power quality issues can be roughly divided into transient state and steady state. The so-called transient power quality is the quality parameters of power system in transient state. The so-called steady power quality is the quality parameters of power system under steady operating mode. 
The essence of the transient power quality is the transient voltage quality problem, or the system impact suffering from external grid interference and internal operating failure. Its main performance indices are voltage pulse, surge, voltage sags and transient voltage interrupt. The effects of the voltage sags are the most common one [8]. The steady state power quality is the main target of power quality monitoring. It includes five main indices: power frequency, voltage deviation, voltage fluctuation and flicker, three-phase voltage unbalance, harmonic voltage and waveform distortion.

Power frequency: frequency is closely linked with active power. The active power absorbed from the grid is dynamic, so the active power between the generator and the user must maintain dynamic homeostasis to keep the bias of voltage and frequency in the normal range. When the active power (or active impact) is severe unbalanced, the grid will bring about frequency deviation.

Voltage deviation: voltage and reactive power are closely linked. In general, the grid user is not purely resistive load, but the $\mathrm{R} / \mathrm{L} / \mathrm{C}$ composite load which generates reactive power in the grid. When the reactive power is larger, it will lead to voltage bias. The capacitive reactive bring about high voltage meanwhile the inductive reactive bring about low voltage.

Voltage fluctuation and flicker: the impact load that load impedance changes quickly will cause reactive power fluctuations. As a result, the voltage amplitude will fluctuate consequently. These voltage fluctuations which less than $30 \mathrm{~Hz}$, especially $8.8 \mathrm{~Hz}$, will cause visual flashing lights, which is the so-called voltage flicker.

Three-phase voltage unbalance: three-phase unbalanced load will produce negative sequence current in the grid, thus generate the three-phase voltage imbalance. The normal voltage unbalance allowable value of the power system common connection point is $2 \%$, short-term may not exceed $4 \%$.

Harmonic voltage and waveform distortion: nonlinear load leads to nonlinear load current. The nonlinear harmonic current can be considered as the superimposing of fundamental current and harmonic current. The harmonic current injected into the grid will produce harmonic voltage, which will result in the voltage waveform distortion after was superimposed on the fundamental voltage.

\section{System Overall Architecture Design}

ZigBee is one of the global standards of communication protocol formulated by the relevant task force under the IEEE 802.15 working group. It is a low-cost, low-power, wireless mesh network standard targeted at wide development of long battery life devices in wireless control and monitoring applications [9-10]. ZigBee devices can transmit data over long distances by passing data through a mesh network of intermediate devices to reach more distant ones. Zigbee builds on the IEEE 802.15.4 standard which defines the physical and MAC layers for low cost and low rate personal area networks. The overview of Zigbee protocol stack was shown in figure 1.

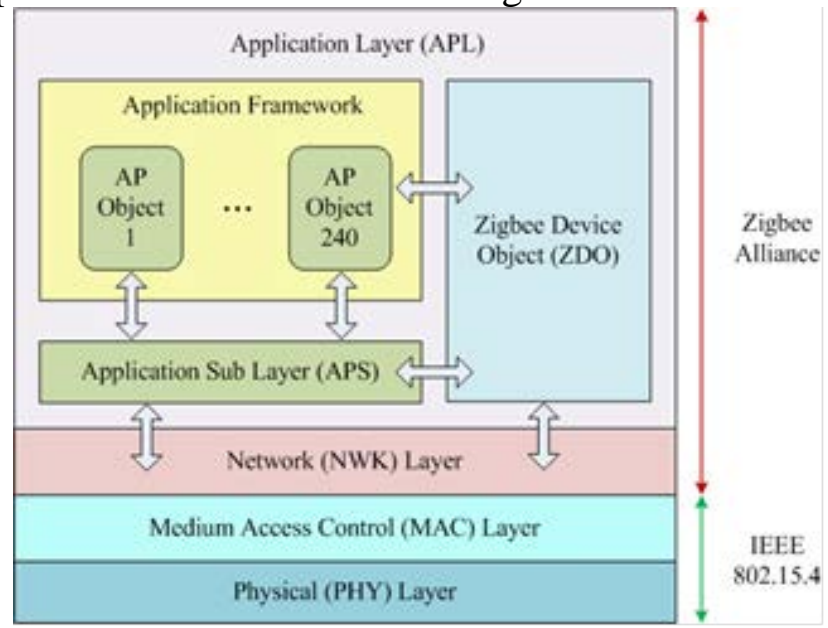

Fig. 1: Zigbee functional layer architecture and protocol stack

Based on those remarkably superiorities of Zigbee technology, Zigbee wireless communication mode was used to replace traditional wired mode in the power quality real-time monitoring system. As shown in figure 2, it consists of data acquisition nodes, sink node and remote monitoring center. 
Data acquisition node and sink node through Zigbee technology compose the WSNs. Sink node and remote monitoring center can carry out wireless data transmission via the Internet or satellite.

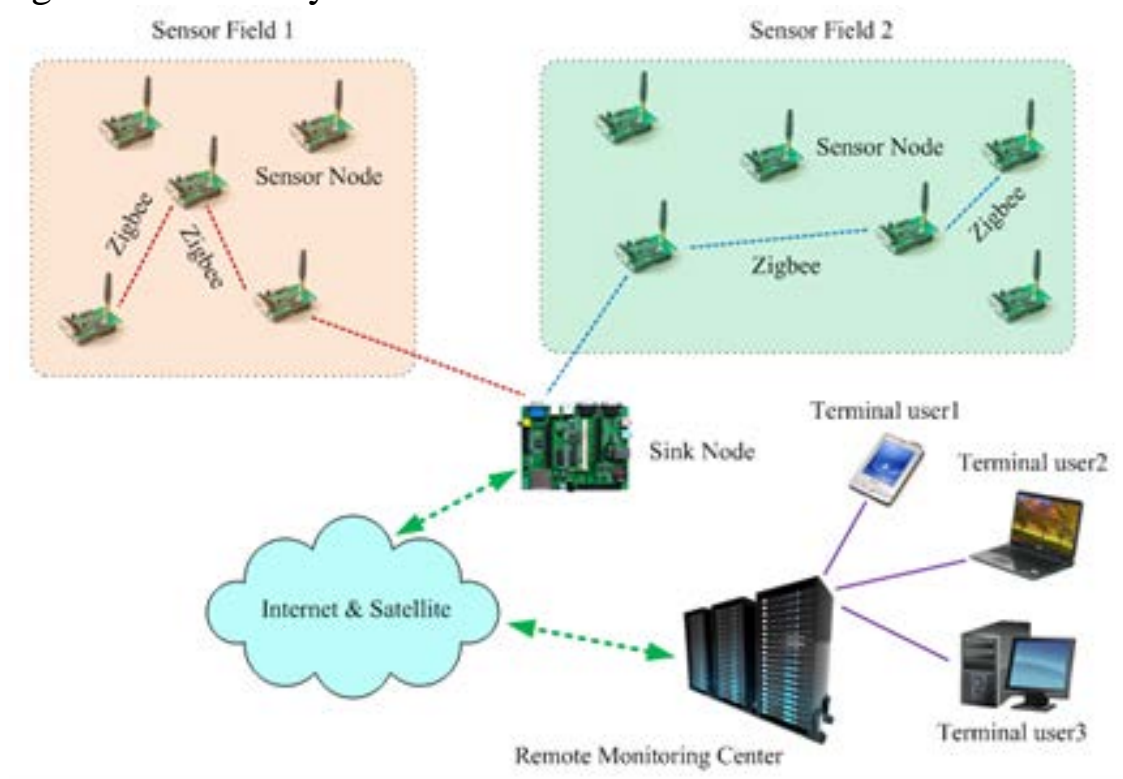

Fig. 2: Overall architecture of real-time monitoring system

\subsection{Data acquisition node}

Data acquisition node consists of sensing unit, processing unit, transceiver, location finding system, mobilizer and power unit, as shown in figure 3 . The voltage and current can be converted into voltage signal which can be received by the DSP. It is mainly responsible for two tasks: transfer power grid parameters from transformer to local aggregation node via Zigbee technology, and set appropriate operating mode according to those received commands.

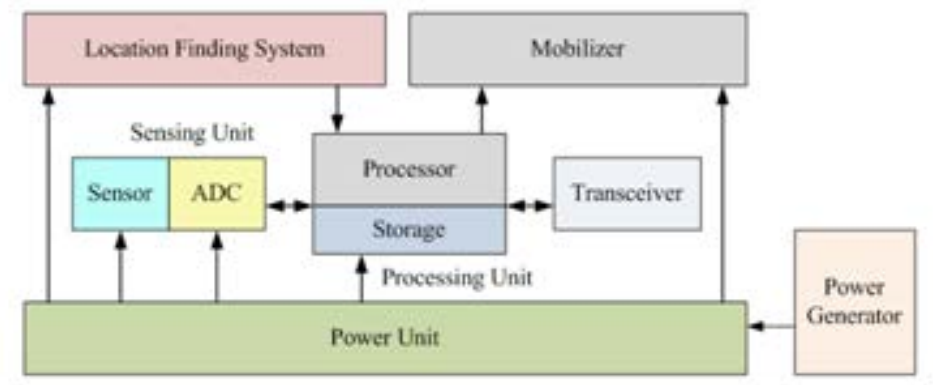

Fig. 3: The component of sensor node

To reasonably deploy these power quality data acquisition nodes, the deployment should cover the main network and all the supply voltage level, and uniformly be distribute in the power consumption area. Moreover, the deployment should be able to commendably meet the adjustment and control requirements of power quality indices and the stringent requirements of voltage-sensitive users.

\subsection{Sink node}

The storage capacity, processing power and communication capability of sink node are relatively stronger than data acquisition. It connects the sensor network and the external network, performs communication protocol conversion between different protocol stacks. Sink node also releases monitoring tasks received from the remote monitoring center, and forwards data to the external network. The main work of sink node is to process the power quality data and compute various technical indices.

The S3C2440A is developed with ARM920T core, $0.13 \mu \mathrm{m}$ CMOS standard cell and a memory complier. Its low-power, simple, elegant and fully static design is particularly suitable for power-sensitive applications. It adopts a new bus architecture known as Advanced Micro controller Bus Architecture (AMBA). By providing a complete set of common system peripherals, the S3C2440A minimizes overall system costs and eliminates the need to configure additional components. Using the ARM9 S3C2440A microcontroller, the local monitoring center was efficiently designed and realized. The circuit of sink node was shown in figure 4. 


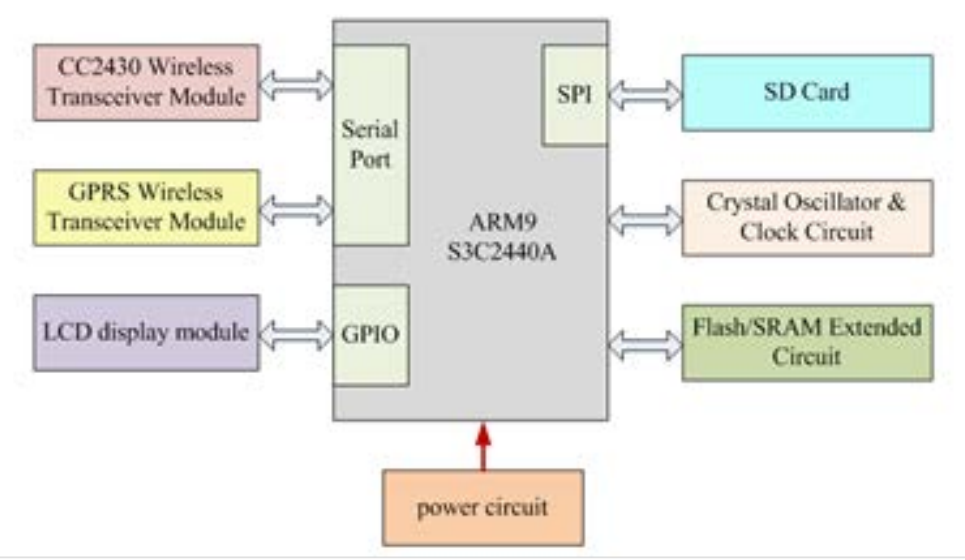

Fig. 4: The circuit of sink node

\subsection{Remote monitoring center}

The remote monitoring center plays an important role of system management. Its main tasks include data analysis, results display, data storage and so on. Those remote users can access the monitoring center server through the Internet platform by the terminal-server form for sharing data and information. Remote monitoring center can communicate simultaneously with multiple WSNs to obtain the power quality parameters in different monitoring areas. Through the effective use of information, the analysis capability, decision-making capability and the intelligence level of the remote monitoring center will be improved greatly. It also provides real-time basis for rational electricity allocation and protection of power systems.

\section{Zigbee-based Network Configuring Process}

Zigbee supports two kinds of physical devices, full function device and reduced function device. The formation of Zigbee-based WSNs consists of two steps, the network initialization and the network access. A sensor node has two ways to join the WSNs, through the sink node or through the existing parent node. The establishment of Zigbee network was launched by sink node. Each node has a unique MAC address by pre-programming method. The network initialization includes two aspects, to determine the initialization parameters and to transmit the selected parameters to those nodes. Node initialization parameters include the operating channel (LogicChannel), PAN ID, node short address (macShortAddress), beacon period (BeaconOrder), super frame activation period (SuperframeOrder) and so on. After being determined, the initialization parameters of will be transmitted to the MAC by calling the MLME-SET primitive and the MLME-START primitive of the MAC layer.

4.1 Join the network through sink node

When the sink node of the Zigbee network was determined, those sensor nodes must first establish connection with the sink node to join the network. The process of sensor nodes join the network was shown in figure 5. To establish connection, FFD nodes need to make a connection request to the sink node. The sink node decides whether to allow the sensor node to connect as receiving its connection request, and then respond to the request according to its situation. When the connection was established between the sensor node and the sink node, they can exchange data. 


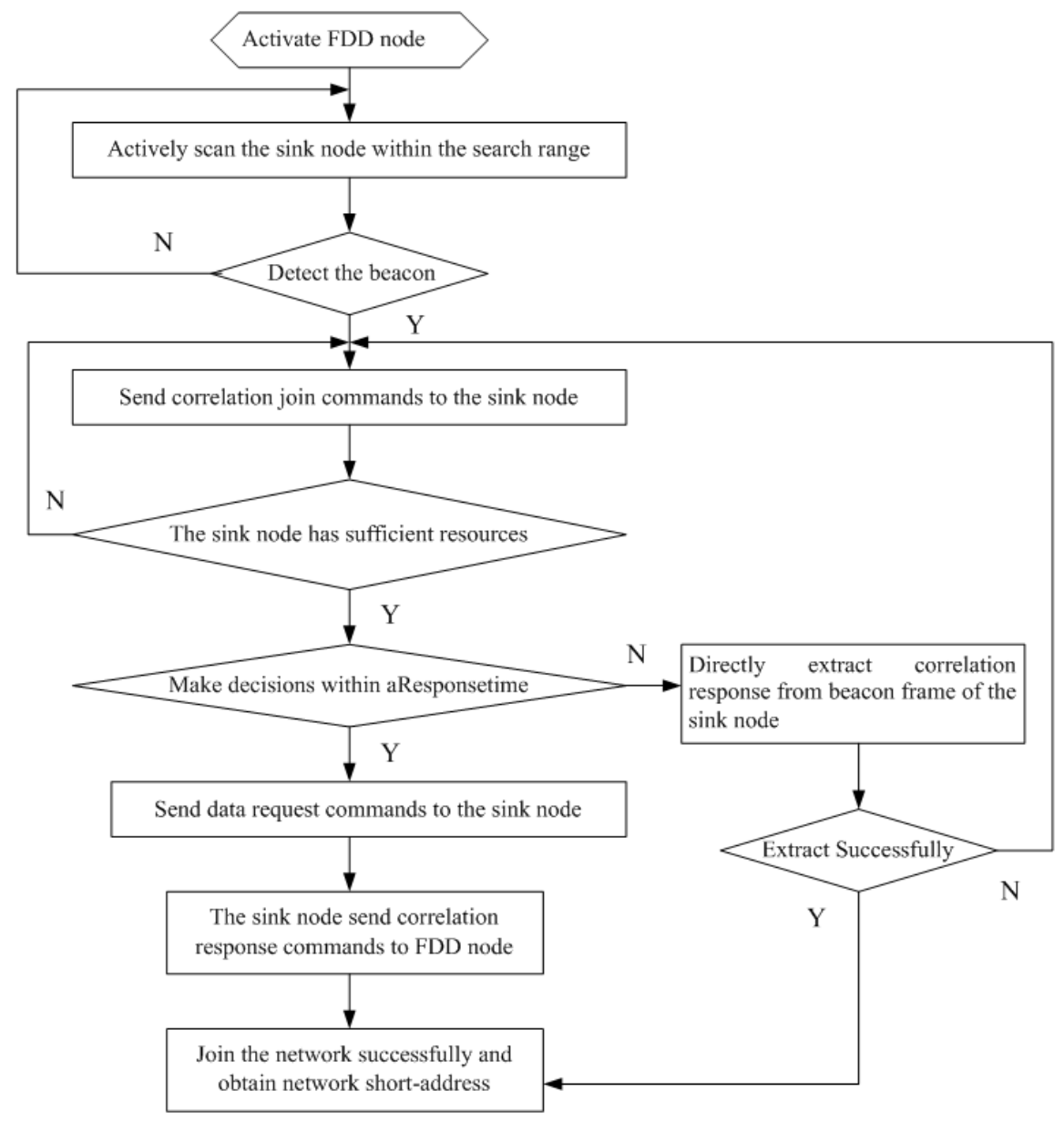

Fig. 5: The workflow of joining the network through sink node

\subsection{Join the network through existing node}

As shown in figure 6, When the sink node successfully associated with those near FFD nodes, the other nodes within the range can make use of those FFD as parent nodes to join the network. In detail, there are two ways to join the network, one is by way of association, and the other is by direct way. The association is the main way to join the network for a new node in the Zigbee network.

For sensor nodes, only those had not joined the network can try to join the WSNs. Those nodes, some had joined the network, but have lost contact with their parent nodes, were called orphan node, while the others are called entirely new node. When a sensor node is orphan node, due to the original parent node information has been stored in its neighbour table, then it can directly send the request of joining the network to its parent node. If the parent node has enough ability to accept its join, the sensor node was directly assigned the previous network address. If its parent node has reached the maximum number of children, that is, the network address has been absolutely assigned, the parent can not authorize it to join. So, the sensor node need look for new network to join. 


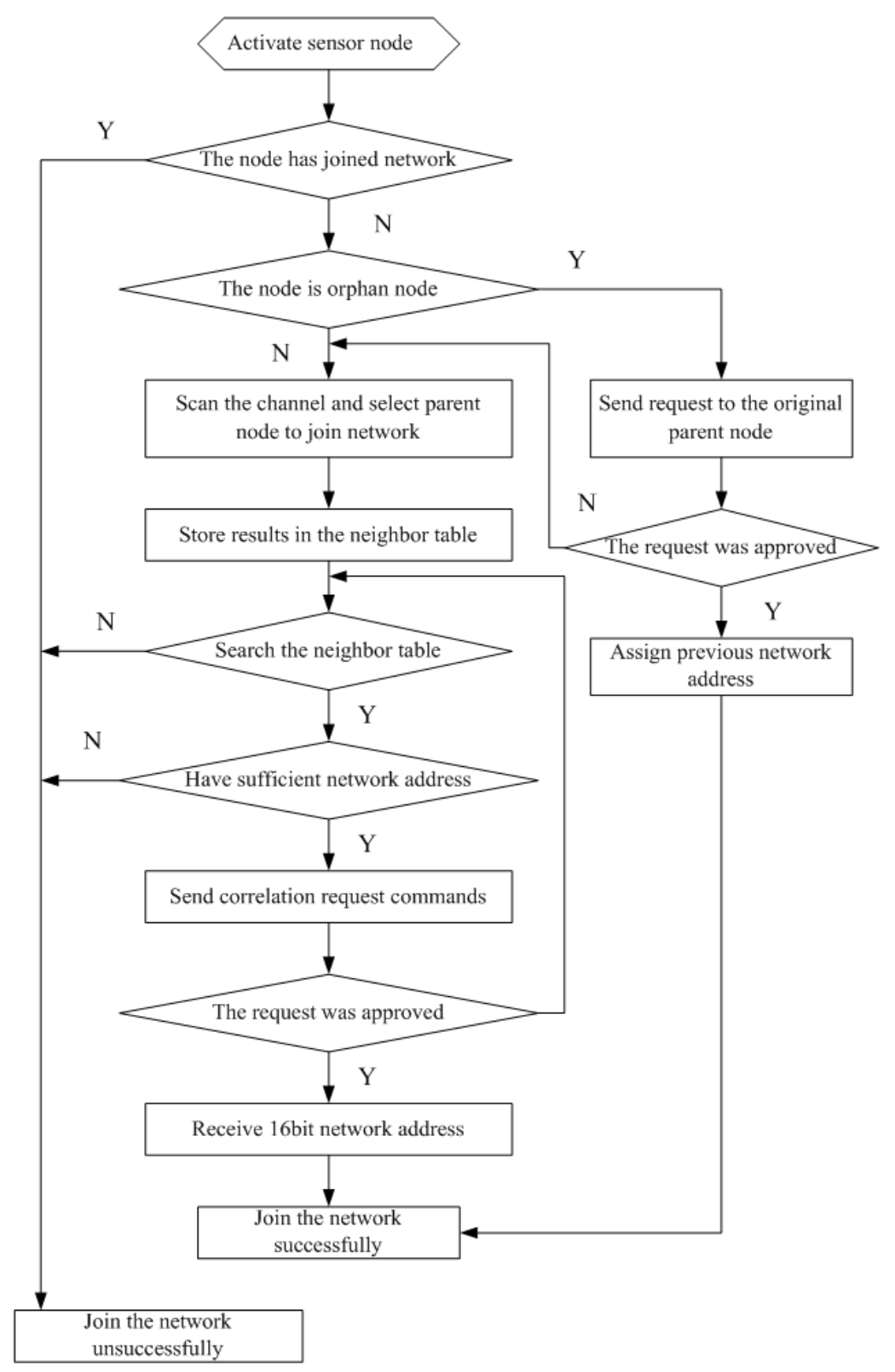

Fig. 6: The workflow of joining the network through existing node

\section{Performance Evaluation}

There are more and more power-sensitive high-tech electrical equipment in industrial enterprises with the raising of the industrial automation level under the new economic situation. Therefore, the real-time power quality monitoring technology proves to be a key link to effectively achieve production efficiency, energy conservation and production safety.

Based on the power quality requirements and the layout of important customer power devices, the real-time Zigbee-based power quality monitoring system was deployed in a mining machinery manufacturing enterprise to verify its performance. Those data acquisition nodes were elaborately deployed to each power devices, for example, those precision machining devices, inverter drives and automated production lines, and so on. The data acquisition node acquire and transmit actual power quality parameters. The sink node is responsible for the collection and storage of the electrical parameters. It also identifies and displays the type of power quality disturbance (PQD). The monitoring system can accurately measure a variety of power quality indices, such as harmonic voltage and waveform distortion, three-phase voltage unbalance, voltage fluctuation and flicker, and so on. The figure 7 shows the PQD recognition results of different sink nodes. 


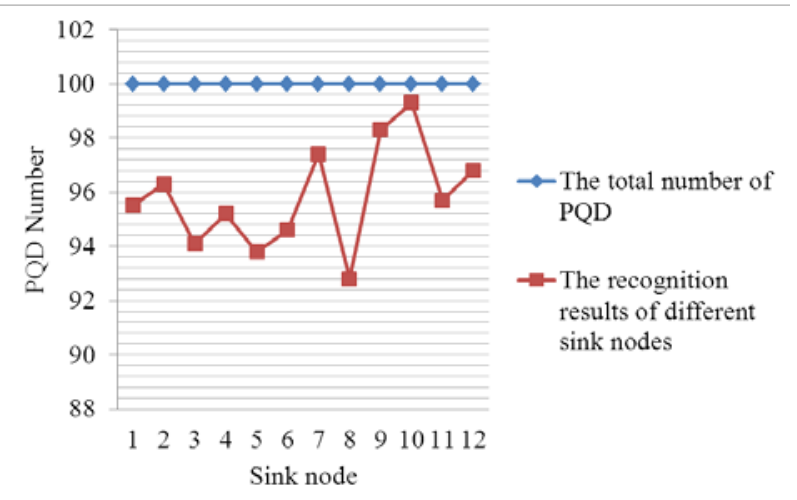

Fig. 7: The PQD recognition results

The distance between the sink node (S3C2440A) and the farthest data acquisition node is about 40 meters. In the actual outdoor point-to-point transmission testing, the straight line transmission distance of wireless module is more than 300 meters. The remote monitoring and management centre locates in the enterprise power management department. Owing to the use of Zigbee wireless transmission module in the real-time power quality monitoring system, it eliminates the need for complex wiring link and saves lots of human and materials costs. On the whole, the features of the system are low cost, high reliability, high capacity and speedy networking process.

\section{Conclusions}

According to the development status of power quality monitoring system, this paper presents a real-time power quality monitoring system based WSNs to safeguard the stable and reliable operation of the grid. It also provide a reliable basis of energy conservation for power consumers. Therefore, the monitoring system has good practical value. The using of WSNs technology in power quality real-time monitoring system provides a good solution to the shortcomings of the previous wired communications. It eliminates the need for complex wiring link and saves lots of construction costs. Due to lots of above-mentioned features, such as high reliability, high capacity and simple networking, the power quality monitoring system provide a valuable concrete practice for the realization of the smart grid.

\section{References}

[1] Angelo Baggini, Handbook of Power Quality. Chichester: John Wiley \& Sons Ltd, 2008.

[2] Eristi H., Yildirim Ö., Eristi B., Demir Y. Automatic recognition system of underlying causes of power quality disturbances based on S-Transform and Extreme Learning Machine, International Journal of Electrical Power and Energy Systems, 61, pp. 553-562, 2014.

[3] Önal Y., Ece D. G., Gerek Ö. N. Hilbert-Huang transform based approach for measurement of voltage flicker magnitude and frequency, Electric Power Components and Systems, 43(2), pp. 167-176, 2015.

[4] Abdoos A. A., Moravej Z., Pazoki M. A hybrid method based on time frequency analysis and artificial intelligence for classification of power quality events, Journal of Intelligent and Fuzzy Systems, 28(3), pp. 1183-1193, 2015.

[5] Nahida Syda, Yasin Sayeed, Rao Kurra Rajasekhara. A study on wireless sensor networks \& designs, International Journal of Multidisciplinary Management Studies, 3(3), pp. 137-146, 2013.

[6] Buratti C., Conti A., Dardari D., Verdone, R. An overview on wireless sensor networks technology and evolution. Sensors, 9, pp. 6869-6896, 2009.

[7] Si-Hun Jo, SeoEun Son, Jung-Wook Park. On improving distortion power quality index in distributed power grids, IEEE Transactions on Smart Grid, 4(1), pp. 586 - 595, 2013.

[8] Tao Lin, Domijan A. On power quality indices and real time measurement, IEEE Transactions on Power Delivery, 20(4), pp. 2552-2562, 2005.

[9] Wei Wang, Guangyu He, Junli Wan. Research on Zigbee wireless communication technology, 2011 International Conference on Electrical and Control Engineering, pp. 1245-1249, Yichang, 2011.

[10] Ramya C. M., Shanmugaraj M., Prabakaran R. Study on ZigBee technology, 2011 3rd International Conference on Electronics Computer Technology, pp. 297-301, Kanyakumari, 2011. 